\title{
The Mathematical Model of the Natural Evolution Law of Cosmic Material Organisms
}

\author{
Sai Chuen Hui \\ Frontier Science Research Institute of Hong Kong Limited
}

\begin{abstract}
The "Goldbach conjecture" is proved by "simultaneous screening of positive and negative sides". And the computational values of "modeling" in various scientific fields coincide with reality, theory, experiment and astronomical observation. The universe is superimposed by the energy of equal positive and negative universe into a coupling strength explosion. After decoupling, the $4 \%$ energy is transformed into "matter" and "biological evolution", and it can justify itself. It is pointed out that the natural evolution of the universe exists in the eternal "law of the universe".
\end{abstract}

Keywords-goldbach's conjecture; positive and negative screening; quantum biology; quantitative change to qualitative change; energy / material world; universal rules

\section{A UNIVERSE OF MATHEMATICS}

Without understanding its language, no one can read the great book of the universe, its language is mathematics Galileo

\section{A. "Goldbach's Conjecture"}

(a) Every Even Number Is The Sum Of Two Odd Prime Numbers.

(b) Each Odd Number Is The Sum Of Three Odd Prime Numbers.

The narrative is concise and difficult to prove. It is known as the Pearl on the crown of mathematics.

\section{B. Positive and Negative one Screening Conclusion}

[Theorem 1] the exact solution formula of Goldbach's conjecture:

$$
\begin{aligned}
& Z_{w}(w)=Z_{p . q}(w)+2 Z_{a}(w) \cdots(2.2)
\end{aligned}
$$

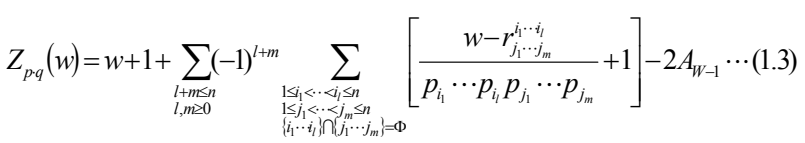

[Theorem 2] any even number as the sum of two odd primes of the odd prime number, the limit value is the total number of odd primes after prescribing and constant not less than 1 .

$$
Z_{w}^{*}(w) \geq\lfloor\pi(\sqrt{w})-1\rfloor+A_{w-1}
$$

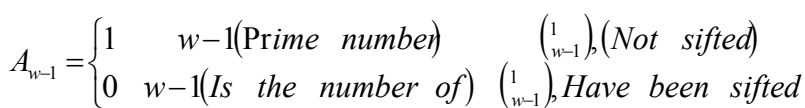

Checking calculation: Taking the minimum value $=6$ of the even number, the limit value

$$
Z_{w}^{*}(w)=[\pi(\sqrt{6})-1]+\left(\begin{array}{l}
1 \\
0
\end{array}\right)=[1-1]+\left(\begin{array}{l}
1 \\
0
\end{array}\right) \geq 1
$$

\section{Goldbach's conjecture}

[Theorem 1-2] confirmed by the United States Intellectual Property Office and 2012.4.4 to the certificate of authorization.

Xiamen University mathematician Li Wenqing (Chen Jingrun teacher) guide the audit results. Opening an equation to solve the two unknowns is an alternative mathematics and is in the ascendant.If we use the formula (1.3) to calculate the "heavy prime number" table, we call it "double key lock cryptography", "biological DNA sequence", "atomic energy meter", "universe $4 \%$ substance" and so on. Can be accurate look up! Implementation of einstein"...... How to set up an aesthetic system that can be expressed strictly by formula "to describe the lifetime wish of the universe with a mathematical formula!"

\section{Six Hexadecimal}

1. "1" is the "concept" of numbers. "1" is not the prime number, is the original minimum unit of things, atomic clock half period cannot show time! Clear concept! Otherwise, a mathematical crisis is inevitable

2. Prime number is the most basic, stable and irreplaceable primitive number of primitive mathematical elements of a thing.

3. Natural integers are divided into even and odd numbers According to the nature of the number, Goldbach conjectures that the mathematical theory is divided into: Each even number is the sum of two odd prime numbers B) every odd number is the sum of three odd prime numbers The minimum common divisor between 2 and 3 is: 2 multiplied by $3=6$ And is the universe matter mathematical "six-ary"; "Six hexadecimal" can prove "twin prime conjectures" and "Liang Dingxiang's conjecture".

4. The ratio of material and cosmic energy to the total mass of $4 \%$ is an example of six calculation.

See the table below 


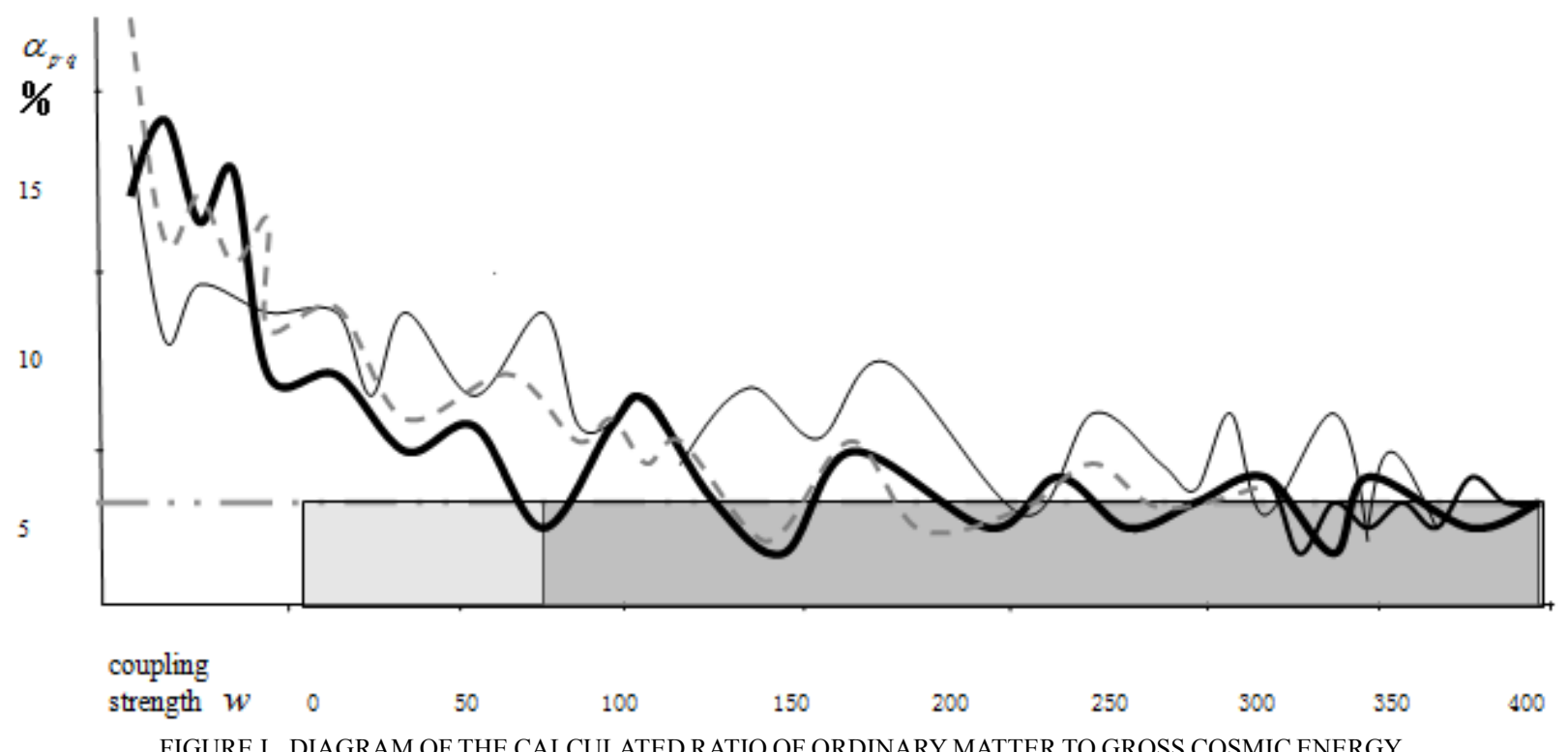

\section{Figure Out}

1. Material atom $\left(\begin{array}{l}p \\ q\end{array}\right)$ The total number of heavy prime numbers accounts for the coupling strength $w$ Ratio: Fine black is the coupling strength $w=6 n n=1,2,3, \ldots$ The curve of the number of atoms (heavy prime)

Green black is the coupling strength $w=6 n+2$ The curve of the number of atoms (heavy prime)

Virtual black is the coupling strength $w=6 n-2$ The curve of the number of atoms (heavy prime)

2. When large even number $w>50$ At a time (yellow region), there are three odd prime numbers of 3.5.7 and the ratio of the substance to the cosmic energy of three quarks $4.6 \%$;

3. When deep black zone is the coupling strength $w \geq 122$ The ratio of average ordinary matter to cosmic energy is coincidental.; $4 \%$ On-line.

(Eight) Six is the most accurate and quickest way to solve dark matter dark energy values

\section{WhAT Is ThE UNIVERSE}

The predecessor of the universe is only the equal positive and negative energy, the algebra and the zero, and the no dimension is the empty state.

\section{A. What is the "Singularity" of the Big Bang?}

There is no matter, no time or space. There are only equal positive and negative energy or the philosophical realm of "unity of opposites" in the universe.

The truth of the "singularity" of the B. Big Bang is
(1) There is no material particle. Where is the volume space of the particle?

(2) There is no big explosion in the universe. What is the energy that makes "the temperature infinitely high"?

(3) There is no physical and material movement. Where is time and space?

Is that the reason? Unbelievable assumptions!

B. The Coupling Explosion Between the Positive and Negative "Cosmic Energy". Decoupling Transmutation into the Material Universe

C. Coupling Strength $w= \pm \sqrt{2 E} \quad E_{\text {Energy }}$

D. Combinatorial Mathematics

Odd number combination-3 A quark composed of particles: an even number of combinations - producing an atom

The gauge field makes the particles transform each other. Now, the new material particles can be accurately calculated by (1.3).

\section{E. Energy Transmutation into Matter}

All things in the universe have the basic law of unity of opposites, and the corresponding mathematics is [positive and negative sifting]: its combination $2^{n}$ It's just the combination of cosmic matter:

a) $\mathrm{A}$ combination of positive and negative 3 different,quantities $n=3 \quad 2^{n}=2^{3}=8$ (hexagram) is the eight state of the particle and the protein eight polymer.

b) The number of 6 combinations of positive and,negative $n=6 \quad 2^{n}=2^{6}=4^{3}=64$ (hexagram) the 64 codons of a creature; 64 basic particles. 
$\left(\begin{array}{l}p \\ q\end{array}\right)$ An atom consists of a proton $p$ (UUD positive energy) and a neutron $q$ (UDD—negative energy). The neutron is an electron and an inverse neutrino, which is an odd prime number. There is no prime factor and can not be decomposed. It is the reason that the hadron can not get out of the quark, but it can only decay.

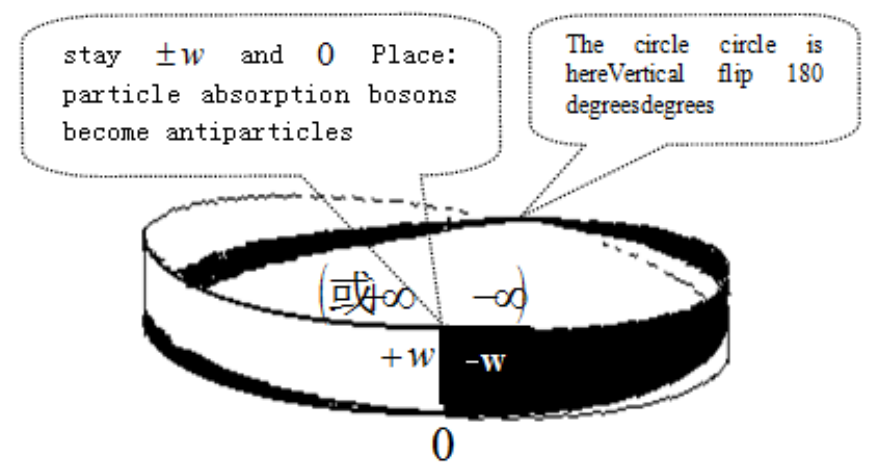

FIGURE II. INVERSE SYMMETRIC ROTATIONAL CYCLE DIAGRAM OF POSITIVE AND NEGATIVE ENERGY UNDER THE CONTROL OF COUPLING STRENGTH EXPLODING UNDER THE TRIGGER OF ANTIMATTER (COSMIC GENE) AND TRANSMUTATION INTO THE MATERIAL UNIVERSE. WITHOUT CHANGING THE MOTION AND ROTATION OF PARTICLES UNDER THE CHARGE CONJUGATION TRANSFORMATION THE COLLISION OF THE TWO PROTONS BECOMES A COLLISION OF TWO ANTIPROTONSTHE COLLISION OF THE TWO PROTONS BECOMES A COLLISI

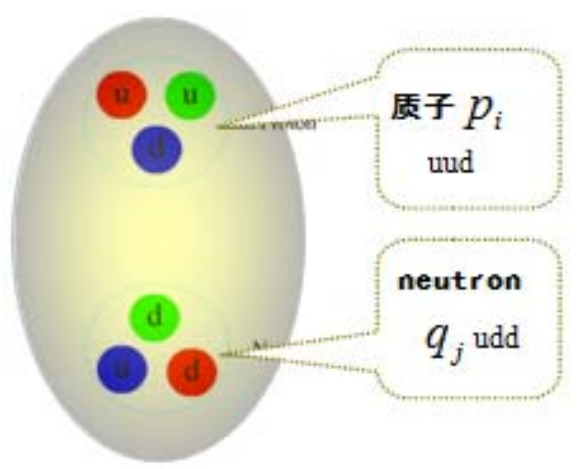

FIGURE III. OTH THE POSITIVE AND THE ANTI ATOMS ARE MADE UP OF PROTONS AND NEUTRONS.THE ELECTRONS IN THE PERIPHERY OF THE ATOM ARE PROVIDED BY NEUTRONS.(NOTE: U QUARKS; D QUARKS)

\section{Material Generation and Evolution}

"So far, scientists do not yet know where the particle mass comes from," Goldbach conjectured: quarks in particles are matter, while wandering quarks are energy. The quark state determines the quality or energy.

\section{A. The Conversion of Energy into the Production of Material Particles and Atoms}

The energy is accelerated in the current accelerator, so that the coupling strength is high enough to decouple the energy of the new particles. Artificial elements such as: 104-111 Rf-Rg

$\begin{array}{lrcccc}\text { Natural numberK } \times 108 & 927.333 & 930.886 & 945.098 & 937.992 \\ 984.181 & 952.204 & 998.393 & 966.416 & & \end{array}$

\begin{tabular}{|c|c|c|c|c|}
\hline Atomic number & 104 & 105 & 106 & 107 \\
\hline 109 & 110 & 111 & & \\
\hline Material element name & 年卢* Rf & 钎杜* Db & 钎喜* Sg & 生波* Bh \\
\hline 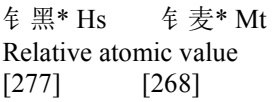 & $\begin{array}{l}\quad \text { 鐽* Ds } \\
\quad[261] \\
{[281]}\end{array}$ & $\begin{array}{c}\text { 錀* Rg } \\
{[262]} \\
{[272]}\end{array}$ & [266] & [264] \\
\hline
\end{tabular}

This is an empirical study of energy transmutation into matter. In other words, "matter is the crystallizer of energy"

Energy is an invisible thing. We can only measure the voltage and quantity of the energy by modern science and technology; who has no ability or ability to measure the three-dimensional volume and age of the energy? Positive and negative energy is equal or not in contact with (Mathematics expressed as 0), only the positive and negative energy superposition coupling strength explosion, decoupling into matter!

\section{B. The Evolution of the Energy Decoupling of the Big Bang into the Material Process}

(1) Under the early weak interaction of the big bang, 3 quarks were gathered and converted into basic particles.mathematical "quality and energy cycle theory": the coupling strength under be concentrated to a proton and neutron in the nucleus (see Figure 8). Mass and energy combinations: divided into so-called odd combinations (particles), even combinations (into atoms) Is the "two wave particle dualism", the fundamental reason of material and energy equivalent.

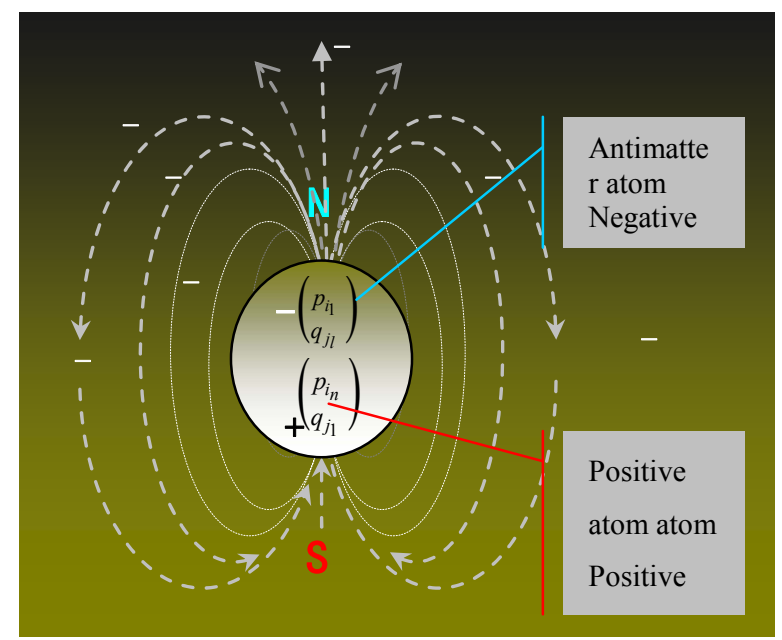

FIGURE IV. THE POLARITY AND MAGNETIC LINE OF FORCE OF ATOMS

Is Schrodinger in the quantum superposition state a paradox of living and giant cats?

\section{Atom - Made up of Positive and Negative Atoms}

Both the positive and the counter atoms are made up of protons and neutrons (see Figure 8) The antimatter atom is the left in (4.3) $\left(\begin{array}{l}p_{i_{1}} \\ q_{j_{L}}\end{array}\right) p-q<0 ;$ Positive atom atom $\left(\begin{array}{c}p_{i_{n}} \\ q_{j_{1}}\end{array}\right) p-q>0 \quad\left[\left(\begin{array}{l}p_{i_{1}} \\ q_{j_{l}}\end{array}\right)+\left(\begin{array}{l}p_{i_{n}} \\ q_{j_{1}}\end{array}\right)\right]$ This is the so-called "atom". What is the difference between a proton and a neutron energy? 
It is the number of peripheral electrons provided by the anti atom. The magnetic field produced by the electronically negative energy is the cause of Universal gravitation (see Figure 9).

\section{Qualitative Change Caused by "Quantitative Change" (See Figure $9 \rightarrow 10$ )}

In the physical universe, antimatter $23 \%$, positive and negative energy account for $73 \%$, and "positive matter" accounts for only 4\%. Antimatter is a category of energy that cannot be decoupled, and antimatter (atom) is the shadow of a positive matter, and is a future cosmological gene. It is the $\mathrm{N}$ pole negative pressure of the magnetic field. So it's hard for us to get in touch with them.

\begin{tabular}{|c|c|}
\hline$p_{i}$ & $q_{w-i}$ \\
\hline$|p|<|q|$ \\
\hline Anti atom \\
Negative pressure \\
The world of energy \\
and light speed \\
No glowing and \\
transparent touch \\
\hline
\end{tabular}

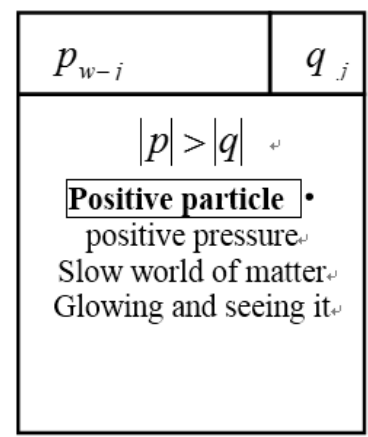

FIGURE V. DIAGRAM OF POSITIVE AND ANTIMATTER DIFFERENCE NOTE: $p$ POSITIVE PARTICle $q$ ANTIPARTICLE ANTIPARTICLE

\section{E. Qualitative Change Caused by "Quantitative Change" (See Figure 9)}

F. The Mechanism of Universal Gravitation (See Figure 11)

The explosion is controlled by strong coupling strength. The anti matter atom decoupling community produced by the atoms of a double material, with the energy of the positive particles and the negative particles, can not counteract each other.

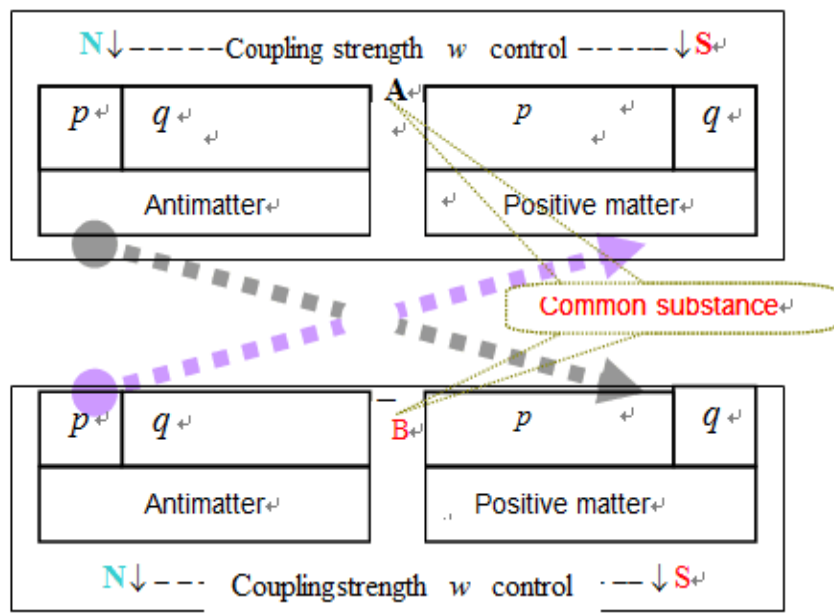

FIGURE VI. UNIVERSAL GRAVITATIONAL INTERACTION MECHANISM

It is the magnetic strength $\mathrm{B}$ of the material itself, the external gravity strength, and the field of the magnetic field (induction).

The Big Bang is in a state of symmetry and antimatter. But astronomical observations show that today's universe is mainly material! Where did the antimatter go? Mathematics shows that the so-called "atom" is the twin community of positive and negative atoms! This section explains the trend of antimatter.

\section{G. Symmetry and Breaking and Mathematical Mathematics}

(1) symmetry is the twin coexistence of a negative atom and a positive atom $\left[\left(\begin{array}{l}p_{i_{1}} \\ q_{j_{2}}\end{array}\right)+\left(\begin{array}{l}p_{i_{2}} \\ n_{j_{1}}\end{array}\right)\right]$ Look up (in 4.3).

$p-q>0$ Positive atom atom
(4.3)Type: $\left(\begin{array}{l}p \\ q\end{array}\right)$ It's a material atom -- one of them $(p)$ Proton positive energy, $\left(q_{q}\right)$ Negative energy of neutron

\section{H. Symmetry Breaking}

It refers to the reduction of variability or symmetry in a certain transformation. That is, a certain characteristic of an object is no longer a constant in a certain transformation, and its symmetry is destroyed, so it is called symmetry breaking.

In coupling strength $w$ Under control, the strong force is in (4.3). $\left(\begin{array}{l}n_{x} \\ q_{j x}\end{array}\right)$ Dark antimatter atom and $\left(\begin{array}{l}p_{i x} \\ n_{x}\end{array}\right)$ Binding of dark positive matter atoms $\left[\left(\begin{array}{l}n_{x} \\ q_{j x}\end{array}\right)+\left(\begin{array}{l}p_{i x} \\ n_{x}\end{array}\right)\right]$ When the "atom": It is negative energy (dark matter). $\left\{\left(n_{x}\right)\right.$ Positive energy, $\left(n_{x}\right)$ Negative energy\} Is the category of energy, only "symmetry breaking"! ( $\left.p_{i x}\right)$ Show only positive particles! 


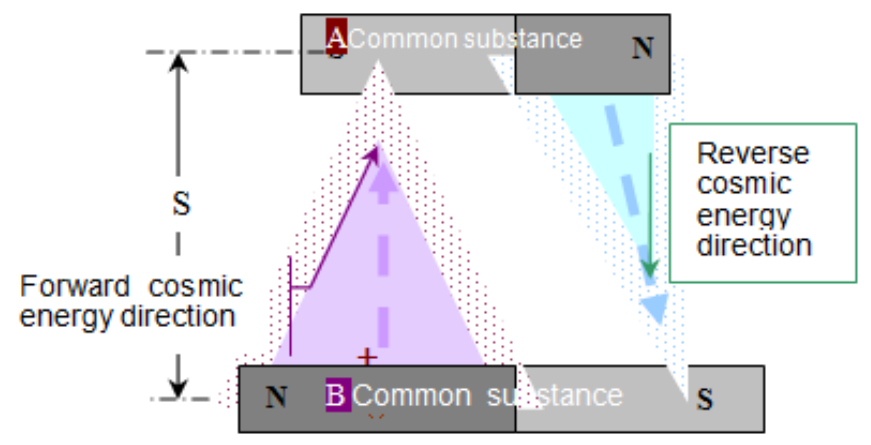

FIGURE VII. THE GRAVITY OF THE MAGNETIC FORCE LINE OF A TWO OBJECT PRODUCES A TWO-WAY COUPLING FORCE

\section{ORIGIN AND EVOLUTION OF LIFE}

A "gene" evolved with biological evolution. The genetic information contained in DNA metabolic evolution is the basis of all modern life functions. In the paleontological world, nucleic acids have a catalytic and genetic function that evolved into a genetic code consisting of four nucleotides. Finally, the optimum number of four bases is obtained.

The formation of organic small molecules, organic macromolecules, amino acids, purines, organic macromolecular proteoglycan, nucleic acids, polysaccharides and polysaccharides are the main components of cytoskeleton. All cell walls and nucleic acids are genetic material.

Through polymerization, the formation of biological macromolecules, self replicating and self selection. Calculation shows that the combination of the material particles and the 64 codons of the biological state of yin and Yang is eight Batu self-organization, replication and mutation. The mathematical correspondence between the DNA double chain complementary sequence and the positive and negative sieves confirms the existence of "natural biologic program software". As long as a person's oligonucleotide primers are primed, that is the initial value ${ }_{j_{1} \cdots j_{m}}^{r_{1} \cdots i_{l}}$, according to (2.3), the DNA sequence of each chromosome can be calculated, which is consistent with the real biological gene encoding rule.

\section{A. Goldbach's Conjecture is Embodied in the Chain of Biological Genes}

An even number $w$ It is the sum of two odd prime numbers $w=p+q$ (the unity of opposites and the combination of yin and Yang) see the picture 13, 14

B. The Basic Combination of All Things in the Universe: $2^{n}$

1. $2^{n}=2^{3}=8$ It is the eight polymer of the protein (the eight state of the particle).

\section{2. $2^{n}=2^{6}=64$ It's the codons 64}

Along as we figure out the primer value of Figure 14 (each person is fixed value), we can calculate all kinds of DNA sequences according to [theorem 1] Goldbach guess's exact solution formula (2.3). (Note: "double key lock password" Chinese and American patent is the live DNA password!)
This is using mathematics to describe biology at a quantum level. Known as "quantum biology".

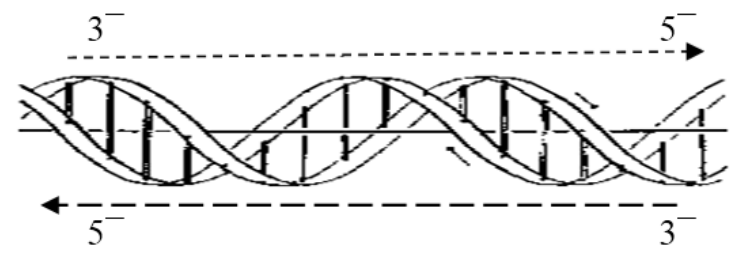

FIGURE VIII. TWO REVERSE PARALLEL POLYNUCLEOTIDE CHAINS AROUND. THE DOUBLE HELIX STRUCTURE WITH A CENTER SHAFT MADE OF THE WINDING

$$
\begin{aligned}
& 3^{\prime} \quad S^{+} \longrightarrow \quad 5^{\prime} \\
& \text { Left hand syndrome positive particle } \\
& \left.S=\left\{\left(\begin{array}{l}
0 \\
w
\end{array}\right)\left(\begin{array}{c}
1 \\
(w-1)
\end{array}\right), \cdots,\left(\begin{array}{l}
p \\
q
\end{array}\right), \cdots, \cdots, \cdots,\left(\begin{array}{c}
x \\
(w-x)
\end{array}\right), \ldots, \cdots, \cdot,\left(\begin{array}{l}
q \\
p
\end{array}\right), \cdots,\left(\begin{array}{c}
w-1 \\
1
\end{array}\right)\left(\begin{array}{l}
w \\
0
\end{array}\right)\right\}\right) \text { Hand card } \\
& \text { Right hand proof antiparticle } \\
& 5^{-} \quad S^{-}
\end{aligned}
$$

The mathematical formula of weak interaction of (1.3) positive and antimatter

C. The Number of Odd Prime Pairs (Base Pairs) $Z_{w}(w)$

The sequence length is the square root of the number of oligonucleotides and $S$ the number of elements of a set $n= \pm \pi(\sqrt{w})$ Corresponding.

If there are "eight polymer" oligonucleotides (a total of 65536) on the chip, the longest sequence length that can be measured is $256 \mathrm{bp}$ (base pair).

even number. The number of all the odd prime number pairs (base pairs) —Exact solution to 1 pages (1.3)

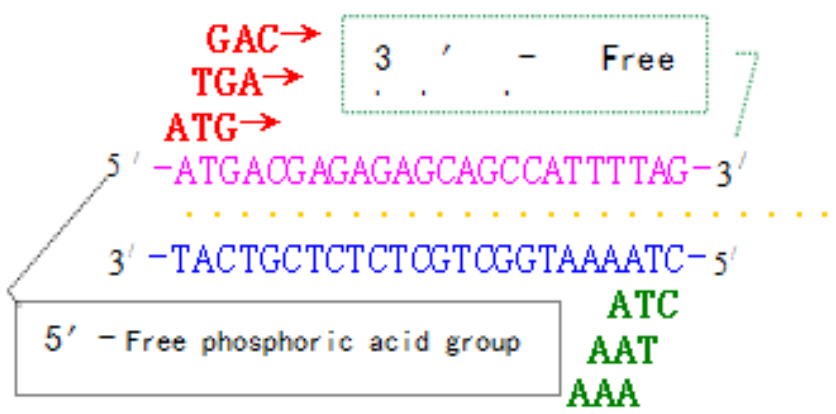

FIGURE IX. CORRESPONDING TO THE THREE YIN SANYANG FIGUE 14 THREE READING FRAME AND TRI GOSSIP. NOTE:

GOLDBACH CONJECTURE THAT EACH ODD NUMBER OF [2] IS THE SUM OF 3 PRIME NUMBERS

\section{Each Odd Number is Equal to the Sum of 3 Primes, Corresponding to Each Codon Consisting of 3 Nucleotides}

The gene of the encoded protein contains the readable frame (ORF), and the analysis complexity is that each DNA sequence has 6 reading frames averaging each. Average $4^{3}=$ 
64 bpOne time.This combination of Yin Yang and Yin and Yang (positive and negative) entity $2^{6}$ Complete anastomosis。For the 64 codons that correspond to the genetic code. DNA can be seen as a character set of four characters: $\Sigma=\{\mathrm{A}, \mathrm{G}, \mathrm{C}, \mathrm{T}\}$ Four decimal code. Base pairing principle detailed: [simple DNA code] patent number: 200810067374.X

The human DNA password is fixed. It is from one person. "Primer value" is the initial value Calculate it by (1.3)!

\section{E. Variation}

In the mathematical formula of Quantum Biology (1.3): the coupling strength of biological DNA code is different from that of 2 units. The difference of Hatching Temperature determines the sex, such as the crocodile, the appearance and personality of children, and the reasons for their differences with their parents.

Only female and male heterosexual combinations will mutate, and cloning will not mutate or decline! The sum of algebra is produced under the condition of "unity of opposites".

\section{COSMIC LAW}

What is the two eternal explanation of the law of Physics

Time is the product of matter plus movement; only mathematics can fully clarify the understanding: "mass energy" is eternal; 2, movement is eternal; it is the intrinsic nature of matter and energy. If the speed of light is the intrinsic property of the photon, the "photon" has only energy and no mass.

\section{A. The Mathematical Conclusion Of Goldbach's Conjecture}

The first unity of opposites in the law of the universe: the combination of yin and Yang

Even conjecture: every even numbe $w(\geq 6)$ It is the sum of two odd prime numbers

1. Superposition of positive and negative energy into coupling strength explosion $4 \%$ energy transmutation into material universe

2. Material atoms $\left[\begin{array}{l}p_{i_{1}} \\ q_{j_{2}}\end{array}\right)+\left(\begin{array}{l}p_{i_{2}} \\ n_{j_{1}}\end{array}\right)$ ]. Anti atom $\left(\begin{array}{l}p_{i_{1}} \\ q_{j_{2}}\end{array}\right)$ Positive atom $\left(\begin{array}{l}p_{i_{2}} \\ n_{j_{1}}\end{array}\right)$ It is the result of opposites and unity The twin community. (Twin community)

3. The breeding inheritance of animals and plants is the positive and anti complementary products of the DNA two chain (see Figure 13).

\section{B. The Basic Particles of the Second Rules - Three Ones}

Odd number conjecture: every odd number $N(\geq 9)$ It is the sum of three odd prime numbers

1. Material particles - the combination of 3 quarks: $p$ proton (uud); q neutron (udd).
2. The biological codons are composed of three nucleotides: each DNA sequence is composed of three forward and three reverse combinations

\section{The Third Rules of the Universe $2^{n}$}

All things in the universe are the transformation of energy! It depends on the coupling strength $w= \pm \sqrt{2 E}$ and the combined elements $n= \pm \pi(\sqrt{w})$. The odd prime number of the "anti matter" (1.3) negative energy of the "antimatter" (anti matter) type is a complete sequence that satisfies $2^{n}$ the need for the conversion of energy into a material double atom. universal gravitation is necessary. All things in the universe are the transformation of energy! It depends on the coupling strength $w= \pm \sqrt{2 E}$ and the combined elements $n= \pm \pi(\sqrt{w})$. The odd prime number of the "anti matter" (1.3) negative energy of the "antimatter" (anti matter) type is a complete sequence that satisfies $2^{n}$ the need for the conversion of energy into a material double atom. $\left[\left(\begin{array}{l}p_{i_{1}} \\ q_{j_{2}}\end{array}\right)+\left(\begin{array}{l}p_{i_{2}} \\ n_{j_{1}}\end{array}\right)\right]$ universal gravitation is necessary.

1. The universe is $n=1 ; 2^{1}=2$ Two combinations of positive and negative energy.

2. Matter is $n=3 ; 2^{3}=8$ matter is the eight heavy state of the particle (protein eight polymer).

3. Biology is $n=6 ; 2^{6}=6464$ codons (64 basic particles).

\section{The Upper Class Base of the Fifth Rule [Index] Growing at the Bottom}

(1) The material universe is both positive and negative energy 2 as the bottom $n$ product, 3 square "eight heavy material particles", and 6 is the biological 64 codon.

(2) In the field of biology, "big fish eat small fish eat shrimp", "law of the jungle" and "biological food chain";

The history of human society: nomadic hunting, fishing, and grain surplus produced a slave society. The stability of agriculture and more grain, the emergence of feudal society; the industrialization of capitalism. Small is the individual, the country, the resources, the interests of the regional war... Will the United States and Russia have a nuclear war? Human beings are living and dying at the crossroads?

\section{E. The Law Of The Fifth Universe -- Cycle And Infinity}

1. The physical universe is running at 0 and infinity (reality is carried out under limited coupling strength), and they are the reciprocal. 180 degrees of particle flipping: charge conjugate transformation... "Antimatter" is a mirror of "positive matter". The mass is exactly the same, and its antiparticle and charge are opposite.

2. Energy converts to material circulation: (1) opposites and unity (2) qualitative change

3. When the substance annihilated, the life body died; the function of the brain disappeared - no soul! 
4. Circulatory immortality - the mating and reproduction of animals, the growth cycle of plants and males and females, the change of social system, and the cycle of quality and energy.

5. The birth and death of the universe

1) Decide on the big bang / black hole struggle, the ultimate black hole to eat out the material

2) The material mass can be $4 \%$ to the line ratio, the material universe is just a moment.

\section{F. The First Driving Force}

The annihilation of a black hole, including light matter, is annihilated into positive and negative energy from both sides. The explosive force that causes the superimposed coupling of the random positive and negative cosmic energy is _ I want to use an animal's instinct to live a good day I dream more about why the natural evolution of the universe is like this

The law of nature is also a two-sided sword.

\section{REFERENCES}

[1] The key to the mysteries of the universe The application of Goldbach's Goldbach conjecture Published by the Hongkong Academy of scientific research. 